\title{
A Novel Method of Electrical Arc Furnace Modeling for Flicker Study
}

\author{
M.A.Golkar ${ }^{*}$ M.Tavakoli Bina ${ }^{*}$ and S.Meschi ${ }^{*}$ \\ * K.N.Toosi University of Technology, Electrical Engineering Department \\ Seyed-Khandan, P. O. Box 16315-1355, (Tehran - Iran) \\ Tel: +98 2122589760 , Fax +982188462066 \\ Emails: magolkar@yahoo.com , sanaz.meschi@gmail.com
}

\begin{abstract}
:
Voltage flicker and harmonics are the power quality problems which are introduced to the power system as a result of nonlinear and stochastic behavior of the arc furnace operation.

To analyze harmonic and flicker generated by an arc furnace, accurate arc furnace models are needed. In this paper, different arc furnace models with different level of complexity are reviewed.

In the first part of this paper a new developed time domain static model based on a piece-wise linear approximation of the V-I characteristics of the arc furnace is presented.

While static arc models are useful for harmonic studies, dynamic models are useful for power quality studies specially the study of voltage flicker.

In the second part of the paper, dynamic model for an arc furnace using MATLAB (Simulink) is presented. In addition to this, the novelty of this simulation technique lies in the fact that the variation of power transmitted to the load by the arc furnace during the cycle of operation is considered, thus making the proposed model more accurate and dependent on the operating conditions of the load.

Finally recommendations are made for the application of some of these models and the accuracy of the presented model to other models from the practical point of view by using MATLAB is shown.
\end{abstract}

Keywords: Electrical Arc Furnace (EAF), Modeling, Power Quality, Flicker, Harmonic

\section{Introduction}

An AC arc furnace is an unbalanced, nonlinear and time varying load, which can cause many problems to the power system quality. An arc furnace load may cause unbalance, harmonics, inter-harmonics and voltage flicker.

To study the behavior of an arc furnace, it is required to develop an accurate three-phase arc furnace model for the purpose of harmonic analysis and flicker compensation. Since the arc melting process is a stationary stochastic process, it is difficult to make an accurate deterministic model for an arc furnace load.
The factors that affect the arc furnace operation are the melting or refining materials, the electrode position, the electrode arm control scheme, and supply system voltage and impedance. Thus, the description of an arc furnace load depends on the following items: arc voltage, arc current and arc length (which is determined by the position of electrode).

In general, the different methods for arc furnace modeling may be classified into the "time domain" and "frequency domain" methods.

The frequency domain analysis method represents the arc voltage and arc current by its harmonic components. Then a power system equivalent circuit for each frequency component is developed and the arc furnace modeled as a voltage source in the same frequency. The total system response is obtained from the superposition of system response in each frequency [1]. One of the requirements of the frequency domain analysis is to have some measured values for the arc voltage and current to obtain the harmonic voltage source model. So this modeling without measurement can not be used. In some references [2]-[4], a harmonic domain solution method of nonlinear differential equation is developed. These equations express the relationship between the arc radius and arc current. These models use some experimental parameters to reflect the arc furnace operation. Because of these experimental and non accurate parameters, these models are not used widely.

Since the electrical arc is a nonlinear and time varying phenomenon, description of its behavior in the time domain is easier than in the frequency domain. Time domain methods are the basic methods for flicker study in electrical arc furnaces. Time domain methods can be classified into V-I Characteristic (VIC), and Equivalent Circuit Methods (ECM).

The ECM methods can be obtained from arc operation; the periodic variation of arc voltage and the resistance that arc shows can be used to develop the arc furnace model [1]. Too much simplification in developing the model may affect the accuracy of the model. 
The VIC methods are based on the V-I characteristic of arc furnace which is derived from the relationship between arc voltage and arc current using the V-I characteristics. This method is widely used for modeling the static and the dynamic operation of EAF.

In the first part of the paper, four different mathematical arc furnace models with different degree of accuracy is obtained from the V-I characteristics. Simulation results are also provided. Then, the dynamic behavior of the reviewed models for flicker simulation is discussed. Finally, a new time domain model for an arc furnace is presented. The new method is studied using MATLAB (Simulink).

\section{Time Domain Static Models}

Figure 1 shows the actual V-I characteristics and its piecewise linear model an arc furnace [1], [5]. The arc ignition voltage $v_{i g}$ and the arc extinction voltage $v_{e x}$ are determined by the arc length during arc furnace operation.

By approximating the actual V-I characteristics of an arc furnace, different time domain models has been derived as follow:

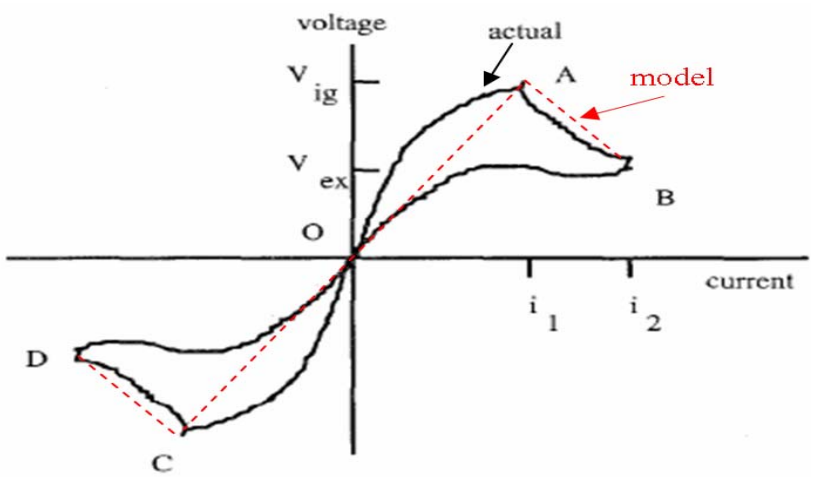

Fig.1. Actual and piece-wise linear approximation of V-I characteristic of an Arc Furnace Load

\section{A. Model1}

The actual arc furnace model can be approximated by two linear equations. Let $R_{1}, R_{2}$ be the slopes of the lines $\mathrm{OA}$ and $\mathrm{AB}$. Thus, in this model the relationship between arc voltage and current for one cycle is expressed as,

$v=\left\{\begin{array}{lc}R_{1} i & -i_{1} \leq i<i_{1} \\ R_{2} i+v_{i g}\left(1-R_{2} / R_{1}\right) & i_{1}<i \leq i_{2} \\ R_{2} i-v_{i g}\left(1-R_{2} / R_{1}\right) & -i_{2} \leq i<-i_{1}\end{array}\right.$

Where

$$
\begin{aligned}
& i_{1}=\frac{v_{i g}}{R_{1}} \\
& i_{2}=\frac{v_{e X}}{R_{2}}-v_{i g}\left(\frac{1}{R_{2}}-\frac{1}{R_{1}}\right)
\end{aligned}
$$

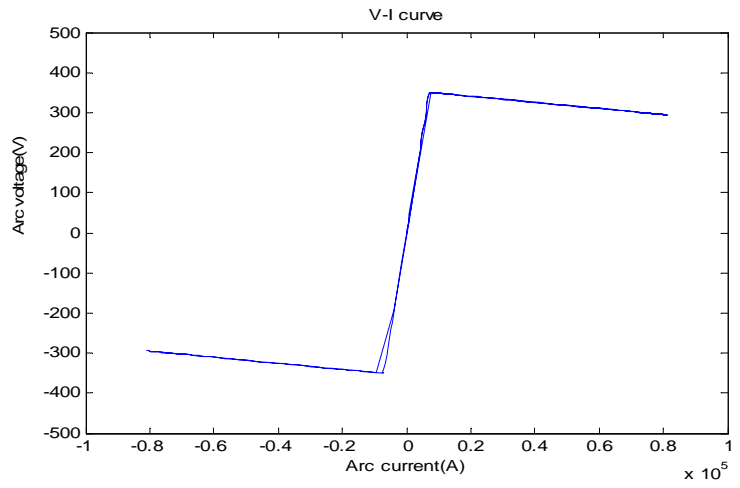

Fig.2.V-I characteristic of model 1

Figure2 shows the simulated V-I characteristic of model 1 using MATLAB (Simulink).

\section{B. Model2}

A more accurate non linear approximation model can be developed by considering part of the characteristics in more details. Figure 3 shows the simulated V-I characteristic of the nonlinear model 2 [1].

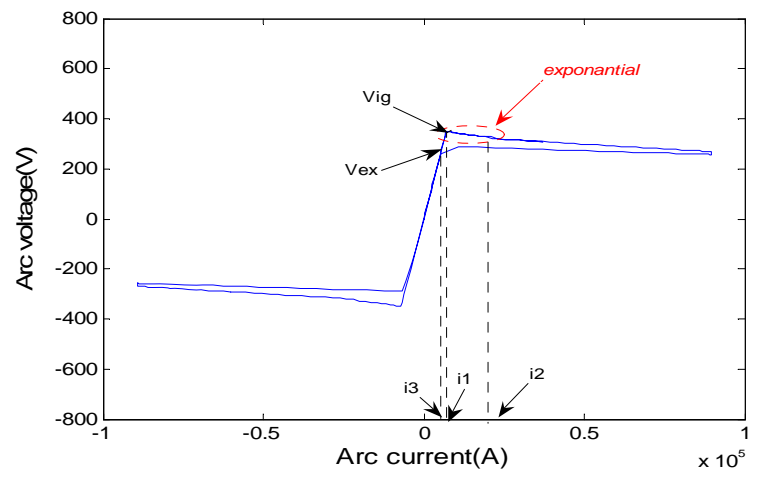

Fig.3. V-I characteristic of model 2

In this model, the arc melting process is divided into three sections. In the first section, the voltage magnitude increases from extinction voltage $-v_{e x}$ to ignition voltage $v_{i g}$. In this part the arc furnace acts as a resistor, and the arc current changes its polarity from $-i_{3}$ to $i_{1}$.The second section is the beginning of the arc melting process. There is a sudden exponential voltage drop across the electrode, thus the arc voltage decreases from $v_{i g}$ to $v_{s t}$, and the arc current has a little increase from $i_{1}$ to $i_{2}$.The third section is the normal arc melting process. The arc 
voltage drops linearly and slowly and smoothly from $v_{s t}$ to $v_{e x}$.

The equations showing these variations are given in 4 .

$$
v= \begin{cases}R_{1} i & \left(-i_{3} \leq i<i_{1}, \text { inc }\right) \text { or } \\ & \left(-i_{1} \leq i<i_{3}, \text { dec }\right) \\ v_{s t}+\left(v_{i g}-v_{s t}\right) \exp \left(\left(i_{1}-i\right) / i_{T}\right) & i_{1} \leq i<i_{2}, \text { inc } \\ v_{s t}+\left(i-i_{2}\right) R_{2} & i \geq i_{2}, \text { inc } \\ v_{e x}+\left(i-i_{3}\right) R_{3} & i \geq i_{3}, \text { dec } \\ -v_{s t}+\left(v_{s t}-v_{i g}\right) \exp \left(\left(i_{1}+i\right) / i_{T}\right) & -i_{2} \leq i<-i_{1}, \text { dec } \\ -v_{s t}+\left(i+i_{2}\right) R_{2} & i<-i_{2}, \text { dec } \\ -v_{e x}+\left(i+i_{3}\right) R_{3} & i<-i_{3}, \text { inc }\end{cases}
$$

Where $R_{1}, R_{2}$ and $R_{3}$ are the corresponding slopes of each section, and

$i_{1}=\frac{v_{i g}}{R_{1}} \quad i_{T}=1.5 i_{1} \quad i_{2}=3 i_{1} \quad i_{3}=\frac{v_{e x}}{R_{1}}$

Figure 3 shows the simulated V-I characteristic of model 2 using MATLAB (Simulink).

\section{Model3}

By examining the actual V-I characteristics of the arc furnace, in general the arc melting process can be divided into three periods. In this modeling some approximations are made according to these three periods, which are explained below: [6], [7]

In the first period, the arc begins to reignite from extinction. When the arc voltage increases to zero, the arc current also reaches its zero crossing point. As the arc voltage reaches to the reignition voltage $v_{i g}$, the equivalent circuit acts as an open circuit. However, a small leakage current exists, which flows through the foamy slag parallel with the arc. The foamy slag is assumed to have a constant resistance $R_{g}$, and the reignition voltage is assumed to be proportional to the arc length.

In the second period, the arc is established. A transient process appears in the voltage waveform at the beginning of arc melting process. The arc voltage drops suddenly from $v_{i g}$ to a constant value $v_{d}$. This process is assumed to be expressed as an exponential function with a time constant $\tau_{1}$.

During the third period, the arc begins to extinguish. The arc voltage continues to drop smoothly, except a sharp change after the arc extinction. This process is also assumed to be represented by an exponential function with a time constant $\tau_{2}$.

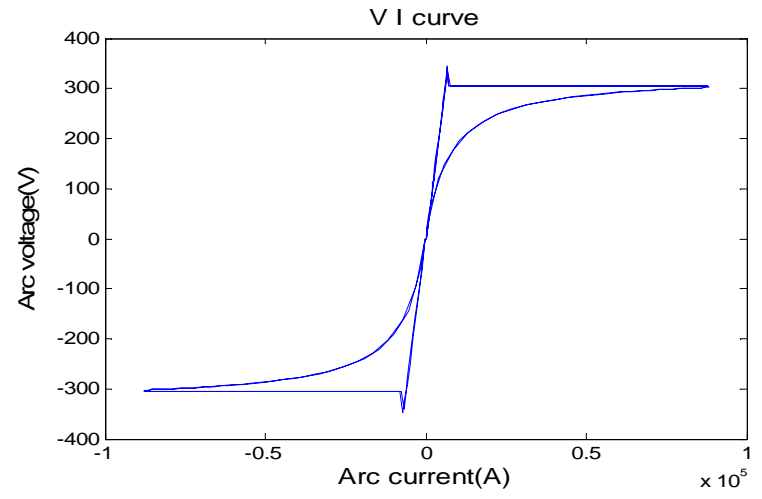

Fig.4. V-I characteristic of model 3

Following the above approximation, the EAF is modeled as a current controlled non-linear resistance and the V-I characteristics is shown in Fig 4. The mathematical representation of EAF are given by (6).

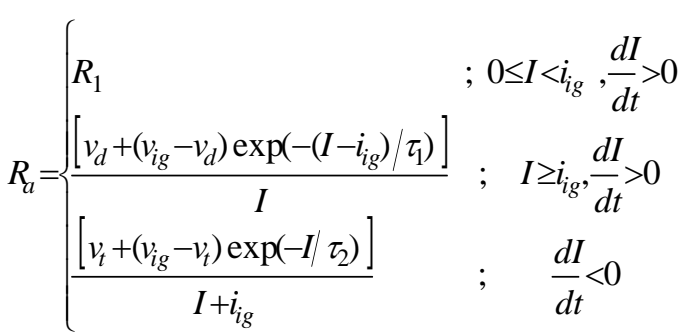

Where

$\begin{array}{ll}I=|i(t)| & v_{i g}=1.15 v_{d} \\ i_{i g}=\frac{v_{i g}}{R_{1}} & v_{t}=\left[\frac{I_{\max }+i_{i g}}{I_{\max }}\right] v_{d}\end{array}$

The current-resistance curve is shows in Fig 5.

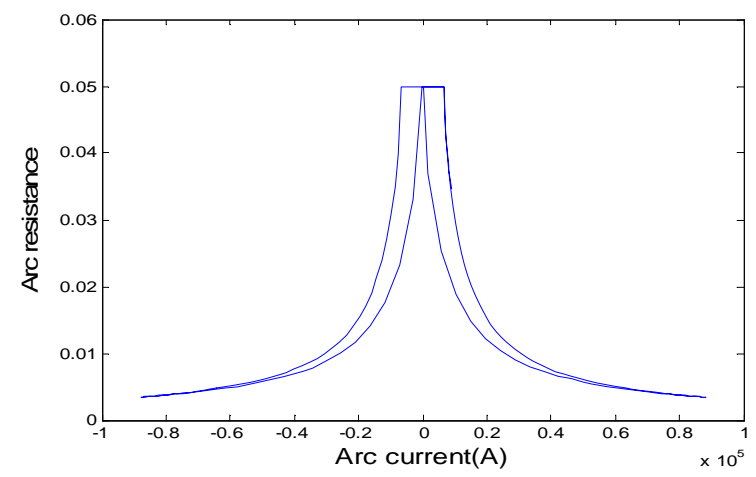

Fig.5. current-resistance curve

\section{Model4}

Another type of modeling and simplification of the actual characteristic is shown in Fig 6. [1], [8]-[12] 


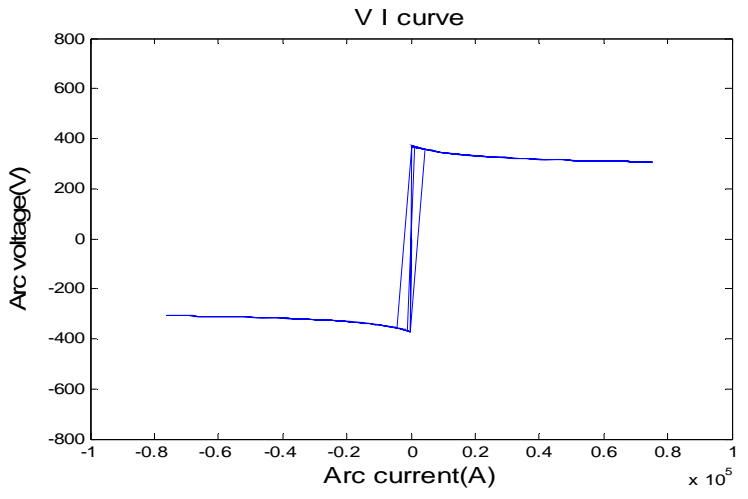

Fig.6. V-I characteristic of model 4

Since the arc voltage changes its polarity very fast, this model neglects the voltage rising time, which results in sudden change in the arc voltage when the arc current is at zero crossing. Thus the perfect cycle of VIC is expressed as,

$$
v=\operatorname{sign}(i)\left[v_{a t}+\frac{C}{D+|i|}\right]
$$

Let " $l$ " be the arc length, A and B are the coefficients from experimental formula, then

$v_{a t}=A+B . l$

Where $v_{a t}$ reflects the arc furnace operating condition.

\section{Dynamic Behavior of Time Domain Models}

Voltage flicker is a stochastic and time varying phenomenon that causes variation in voltage RMS in the frequency range (0.5-25 HZ).

To analyze flicker generated by an arc furnace, the dynamic arc furnace model is needed. To generate the dynamic behavior of the arc furnace using different models, the slop of the V-I curve in figure 1 should be changed as a sinusoidal function.

In models 1, 2, and 3 the dynamic load models considers periodic change of the arc resistance about the value $R_{1}$ given for each model. The arc resistance in the case of sinusoidal variation is defined as:

$R_{1}(t)=R_{1}\left(1+m \sin \left(\omega_{f} t\right)\right)$

Where $R_{1}$ is the constant resistance of arc furnace when the arc is off and EAF acts as an open circuit, $\omega_{f}$ is the

flicker frequency, and $m$ is the modulation coefficient.

In model 4, the dynamic load model is affected by the voltage $v_{a t}$ in equation 9 , so voltage $v_{a t}$ can be considered as:

$v_{a t}(t)=v_{a t}\left(1+m \sin \left(\omega_{f} t\right)\right)$

\section{Purposed Method for Simulate Flicker by EAF}

In the models presented, the arc furnace dynamic model is generated by changing R1 (say R1 modulation). Referring to the VIC of arc furnace, the R1 is associated with the low current period of the furnace cycle, though R2 is the period which is the active part of the cycle and more current is passing through the electrodes so more flicker is expected.

If we use R2 modulation to generate the dynamic response of the furnace, more flickers is generated and the results seem to be closer to the real one.

As more power is taken by the furnace in part $A B$ of the VIC, more flickers is expected and using R1 modulation leads us to a less generated flicker case which is not correct.

The most flickers are generated when the length of the arc is changing and this period is in part $A B$ in the characteristic.

So the dynamic behavior of the furnace can be generated by R2 modulation as:

$R_{2}(t)=R_{2}\left(1+m \sin \left(\omega_{f} t\right)\right)$

\section{Simulation Results}

To compare the different arc furnace models, a simple arc furnace system is studied in single phase. The system configuration is shown in Fig 7 .

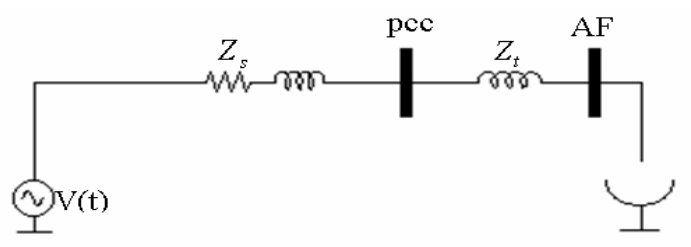

Fig.7. Test arc furnace system diagram

In figure7, the system impedance is represented as $Z_{s}$, bus PCC represents the point of common coupling, and bus AF is the low voltage side of the transformer whose impedance is given as $Z_{t}$.

Simulation diagram is shown in Fig 8 and the system parameters and the parameters of each model are presented in Table I. 
TABLE I. System and arc furnace model parameters

\begin{tabular}{|c|ll|}
\hline items & \multicolumn{2}{|c|}{ Parameters } \\
\hline \multirow{3}{*}{ system } & $V=566 \mathrm{~V} \quad f=60 \mathrm{HZ}$ \\
& $Z_{s}=0.0528+j 0.468 \mathrm{~m} \Omega$ \\
& $Z_{t}=0.3366+j 3.22 \mathrm{~m} \Omega$ \\
\hline \multirow{3}{*}{ Model 1 } & $v_{i g}=350.75 \mathrm{~V}$ & $R_{1}=.05 \Omega$ \\
& $i_{1}=7015 \mathrm{~A}$ & $v_{e x}=289.75 \mathrm{~V}$ \\
& $R_{2}=-0.76 \mathrm{~m} \Omega$ & $i_{2}=87.278 \mathrm{kA}$ \\
\hline \multirow{3}{*}{ Model 2 } & $v_{i g}=350.75 \mathrm{~V}$ & $v_{e x}=289.75 \mathrm{~V}$ \\
& $v_{s t}=320.75 \mathrm{v}$ & $R_{1}=.05 \Omega$ \\
& $R_{2}=-0.76 \mathrm{~m} \Omega$ & $R_{3}=-0.39 \mathrm{~m} \Omega$ \\
\hline \multirow{2}{*}{ Model 3 } & $v_{i g}=350.75 \mathrm{~V}$ & $I_{\text {max }}=100 \mathrm{kA}$ \\
& $\tau_{1}=0.01 \mathrm{sec}$ & $\tau_{2}=0.02 \mathrm{sec}$ \\
\hline \multirow{2}{*}{ Model 4 } & $v_{a t}=289.75 \mathrm{~V}$ & $C=1.68 \mathrm{MW}$ \\
& $D=20.65 \mathrm{kA}$ & \\
\hline
\end{tabular}

Using the parameters shown in table 1, the four different arc furnace models are studied, and the results are shown in Fig 9-12 respectively. The arc voltage, voltage at PCC and arc current are shown in each figure. All the current waveforms in the figures are scaled to 200, in order to match the voltage waveform. The harmonic component of arc voltage, arc current and PCC voltage for each model is given in table II.

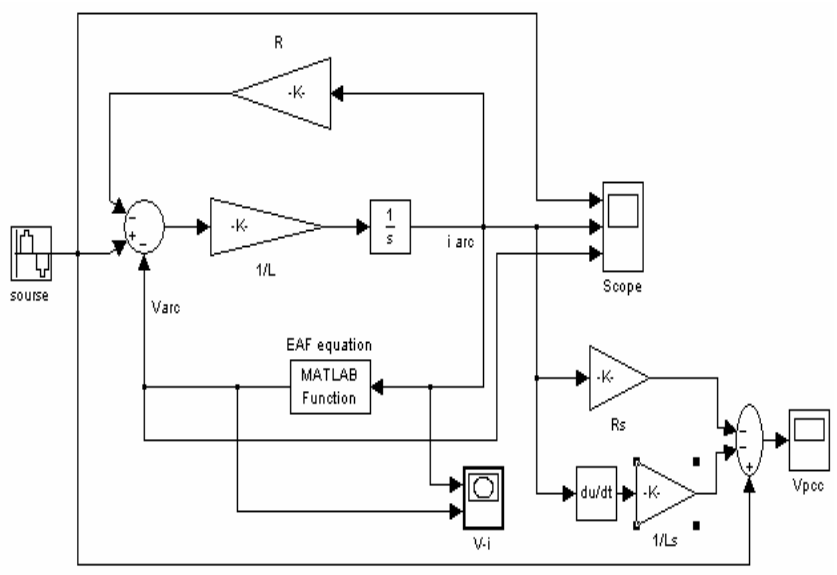

Fig.8. MATLAB diagram of EAF

To determine the flicker introduced by EAF at PCC bus, the dynamic model of electrical arc furnace must be studied. The dynamic behavior is based on the arc length variations. The nonlinear and time varying characteristic of the arc length cause changes in arc resistance and so the slope of the V-I characteristic.
TABLE II. Comparison of the harmonic component of different models

\begin{tabular}{|c|c|c|c|c|c|}
\hline Item & Harmonic & $\begin{array}{c}\text { Model } \\
1\end{array}$ & $\begin{array}{l}\text { Model } \\
2\end{array}$ & $\begin{array}{c}\text { Model } \\
3\end{array}$ & $\begin{array}{l}\text { Model } \\
4\end{array}$ \\
\hline \multirow{6}{*}{ 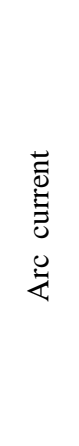 } & Fund(KA) & 72.226 & 81.824 & 80.727 & 65.56 \\
\hline & 3 rd (\%) & 0.1846 & 0.15068 & 0.13644 & 0.20636 \\
\hline & 5th (\%) & .06081 & .05095 & .043556 & .07135 \\
\hline & 7th (\%) & .02577 & .02125 & .017429 & .0319707 \\
\hline & 9th (\%) & .01221 & .012324 & .0091264 & .0162088 \\
\hline & 11th (\%) & .00716 & .009785 & .0072054 & .0095027 \\
\hline \multirow{6}{*}{ 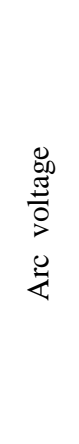 } & Fund(V) & 398.11 & 362.7 & 391.3432 & 407.1365 \\
\hline & 3 rd (\%) & 0.3571 & 0.35842 & 0.301321 & 0.364177 \\
\hline & 5th (\%) & 0.1954 & 0.21053 & 0.158682 & 0.213650 \\
\hline & 7th (\%) & 0.1247 & 0.1412 & .09551 & 0.147125 \\
\hline & 9th (\%) & .08625 & 0.10191 & .060519 & 0.11298 \\
\hline & 11th (\%) & .06259 & .07885 & .03832 & .09348 \\
\hline \multirow{6}{*}{ 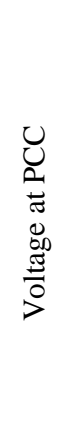 } & Fund(V) & 539.5 & 534.738 & 536.387 & 542.9 \\
\hline & 3 rd (\%) & .03349 & .030949 & .02805 & .034536 \\
\hline & 5th (\%) & .01853 & .018317 & .015553 & .0204236 \\
\hline & 7th (\%) & .01182 & .012202 & .010111 & .014127 \\
\hline & 9th (\%) & .00811 & .008688 & .007121 & .01083 \\
\hline & 11th (\%) & .00581 & .006709 & .005253 & .008902 \\
\hline
\end{tabular}

The main reason to choose an appropriate modulation factor is to generate acceptable flicker and at the same time limit the variations in the slop of the V-I characteristics.

Figures 13-16 shows the dynamic V-I characteristic. The measurement of voltage flicker involves the derivation of system RMS voltage variation and the frequency at which the variation occurs, so the percent voltage flicker ( $\frac{\Delta V}{V} \%$ )and flicker frequency are important [13]. The mathematical relationships and definitions for cyclic voltage flicker are described in reference [13].

From the calculations, $\frac{\Delta V}{V} \%$ at 10 hertz for 4 models is represented in Table III. 


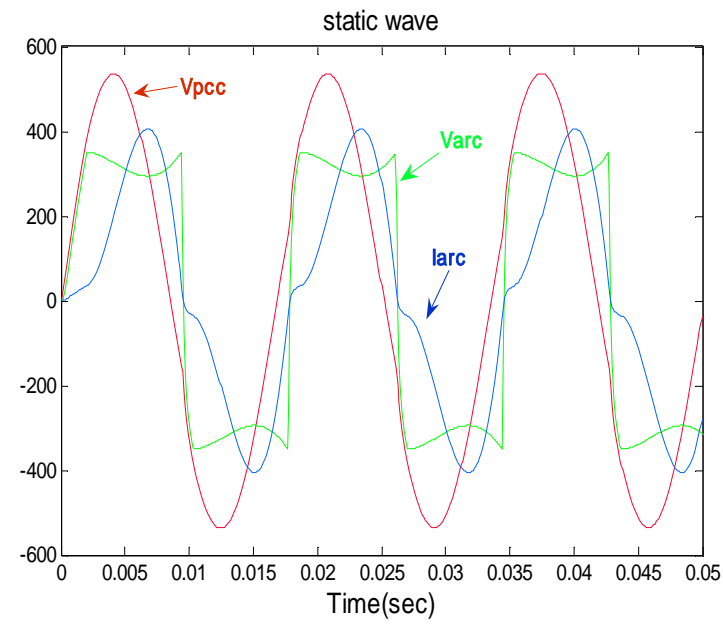

Fig.9. Voltage and current waveform from model 1in static state

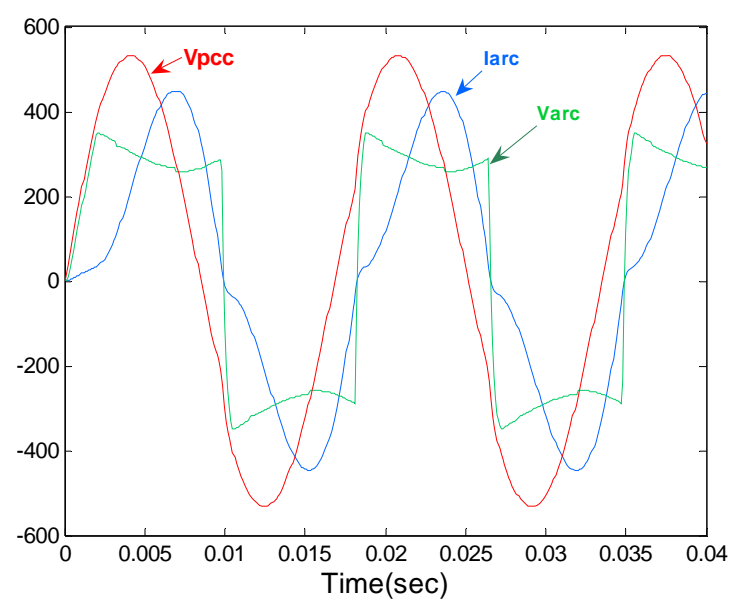

Fig.10. voltage and current waveform from model 2in static state

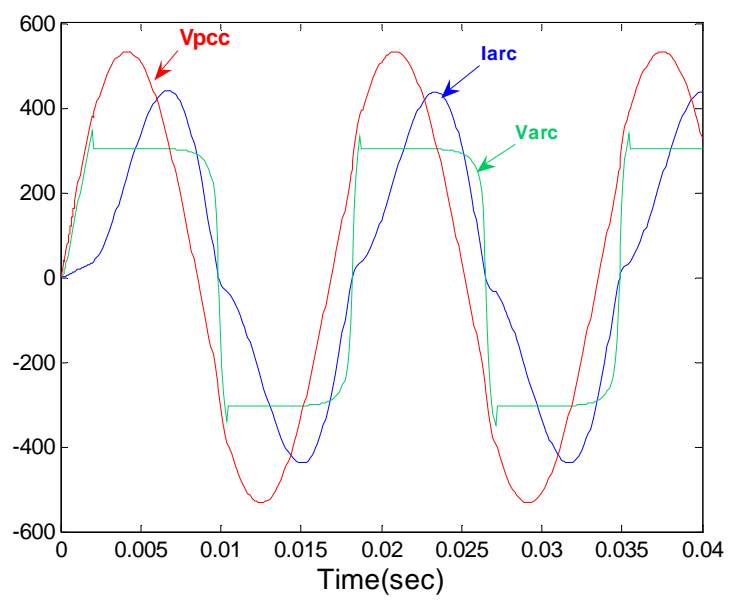

Fig.11. Voltage and current waveform from model 3 in static state

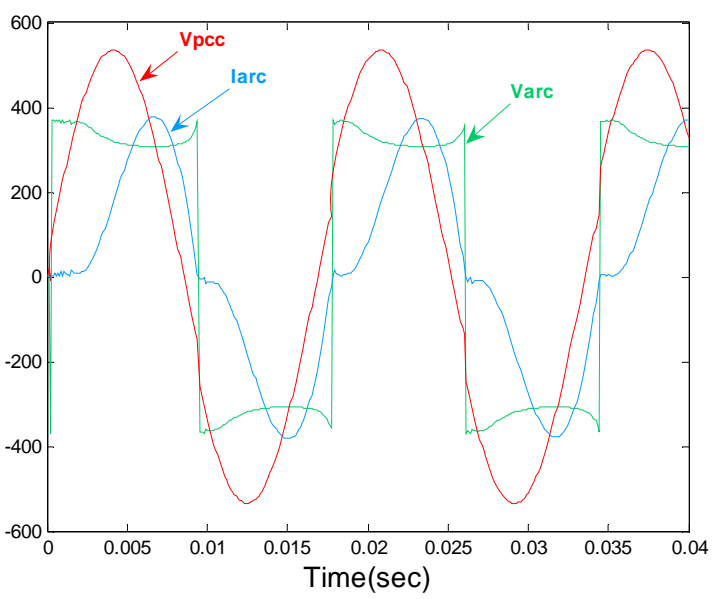

Fig.12. Voltage and current waveform from model 4 in static state

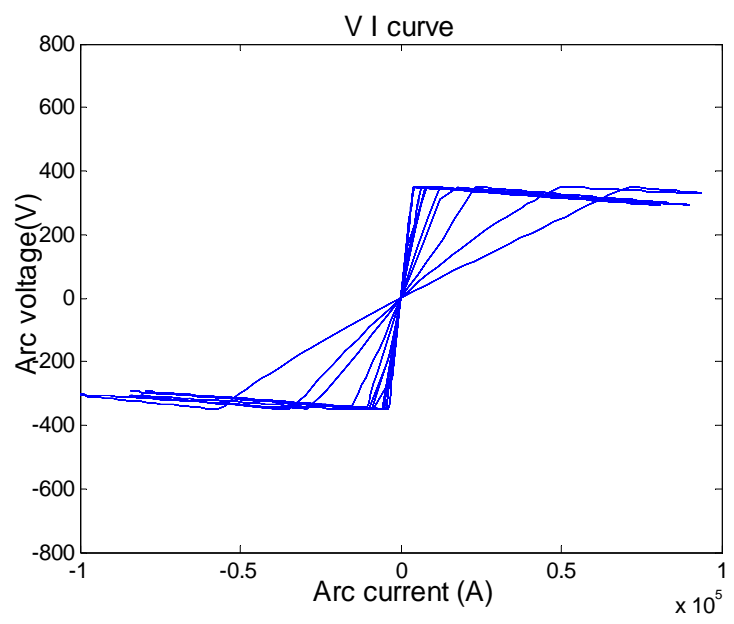

Fig.13. Dynamic V_I characteristic of model 1

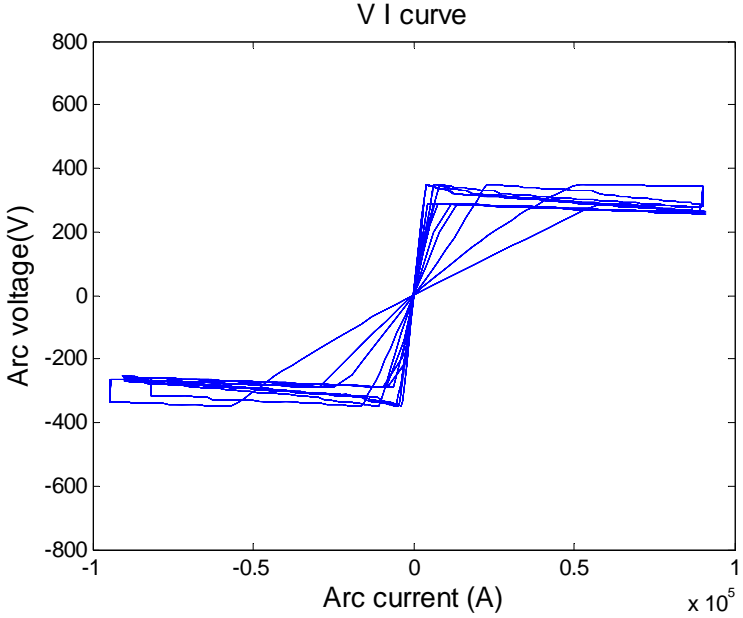

Fig.14. Dynamic V_I characteristic of model 2 


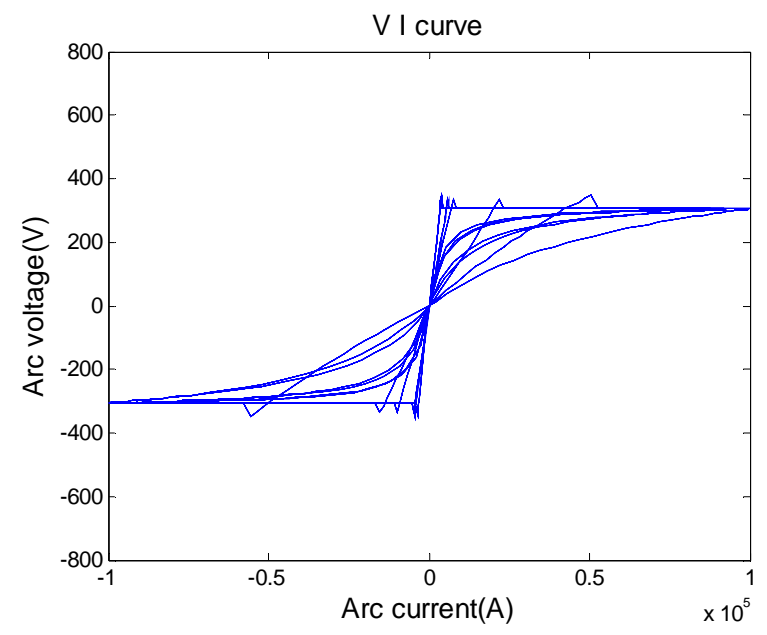

Fig.15. Dynamic V_I characteristic of model 3

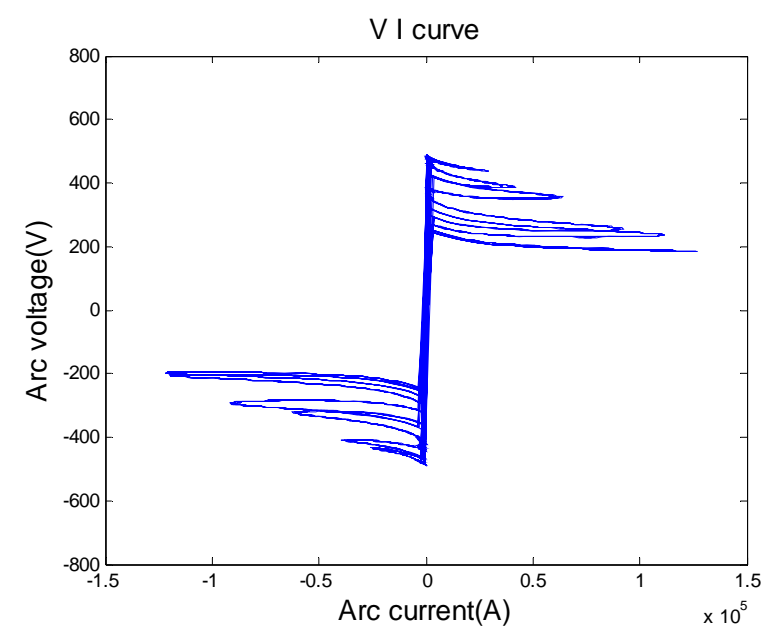

Fig.16. Dynamic V_I characteristic of model 4

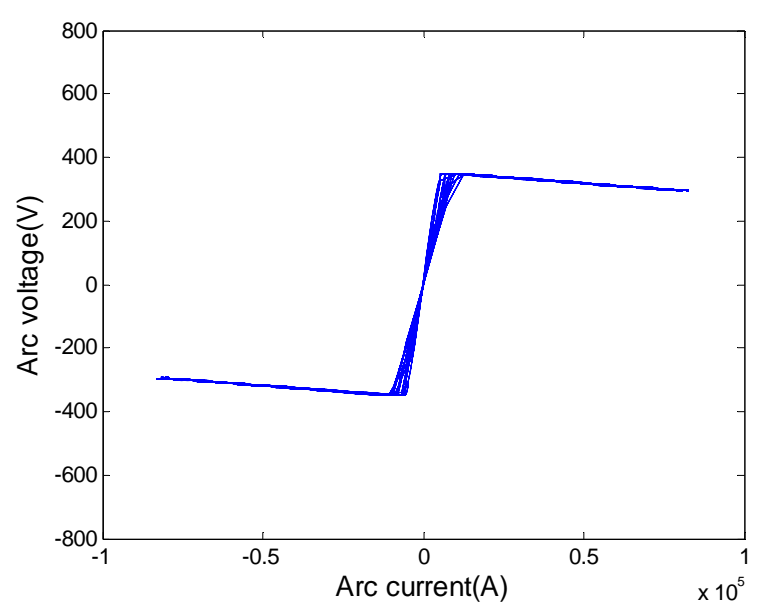

Fig.17.V-I characteristic from model 2 with $R_{1}$ modulation

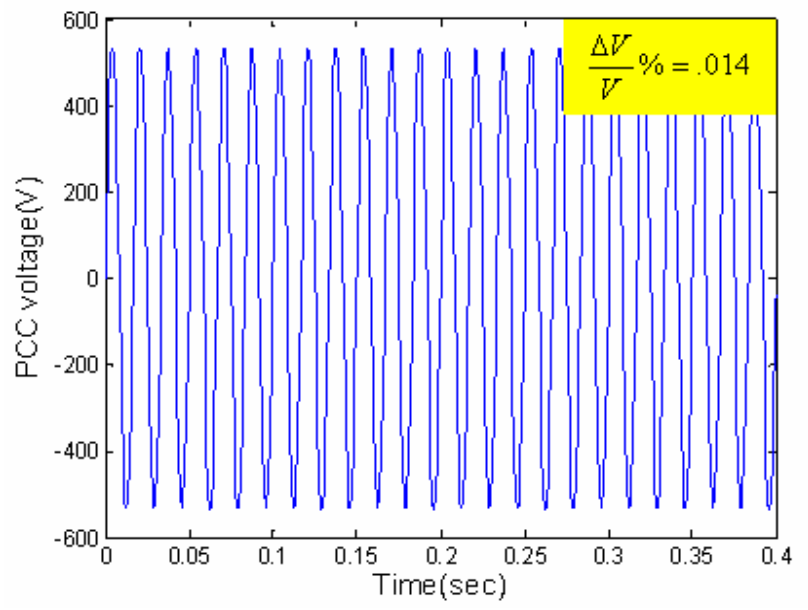

Fig.18. PCC voltage with $\mathrm{m}=0.3$ for $R_{1}$

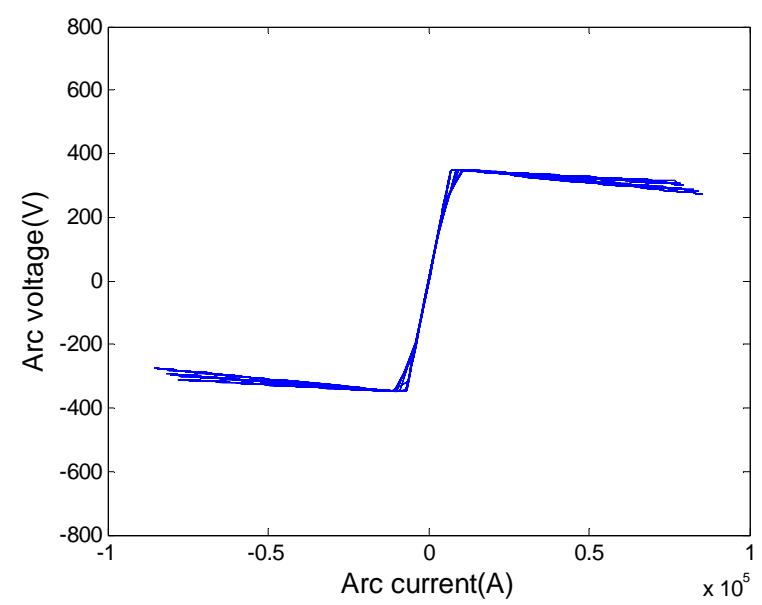

Fig. 19.V-I characteristic from model 2 with $R_{2}$ modulation

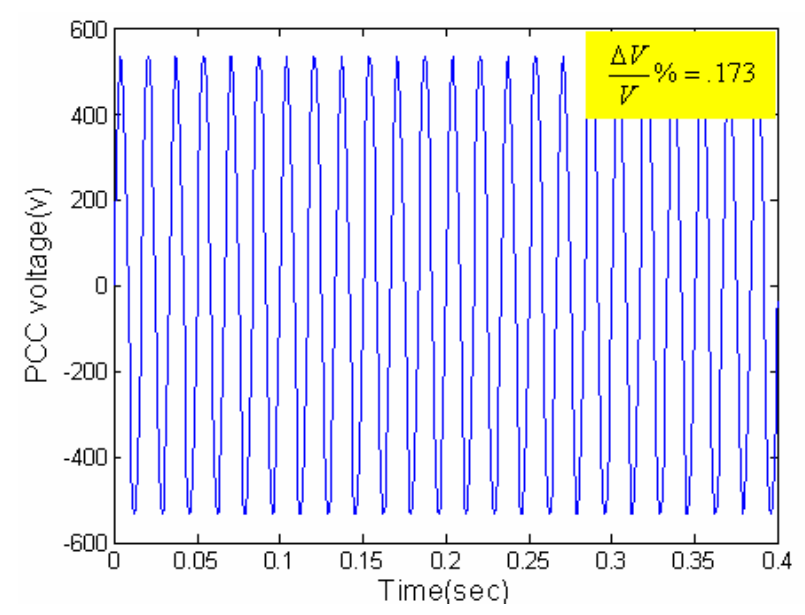

Fig.20. PCC voltage with $\mathrm{m}=0.3$ for $R_{2}$ 
Table III. $\frac{\Delta V}{V} \%$ at $10 \mathrm{~Hz}$ for sinusoidal variation of arc

\begin{tabular}{|c|c|}
\hline Model type & $\frac{\Delta V}{V} \%$ \\
\hline Model 1 & $0.2207 \%$ \\
\hline Model2 & $0.5956 \%$ \\
\hline Model3 & $0.00887 \%$ \\
\hline Model 4 & $3.047 \%$ \\
\hline
\end{tabular}

\section{Conclusion}

Arc furnace is usually modeled in frequency and time domain. Modeling in frequency domain needs some measurements that in most cases is not provided that is why time domain modeling is more favorable.

In this study, different models for arc furnace in the time domain are reviewed. The modeling is based on V-I characteristic approximation. Four major models for flicker evaluation of arc furnace are studied in this paper.

In the models 1,2 , and 4 arc furnace is modeled as a current controlled voltage source, however, in the model 3 the arc furnace has been modeled as a current controlled nonlinear resistance.

In whole models mathematical relationship for perfect performance cycle is obtained with assumption that V-I curve is symmetric with respect to origin of axis. So all the models only generates odd harmonics. However actual arc furnace generates even harmonics.

Referring to Fig1, the actual V-I characteristic of arc furnace has three parts. In the first period, the arc begins to reignite from extinction. When the arc voltage reaches to the reignition voltage $v_{i g}$, the equivalent circuit acts as an open circuit. In the second period, the arc is established and the arc the voltage drops from $v_{i g}$ to $v_{e x}$ increase the electrical conductivity of the arc (AB path). During the third part, the arc begins to extinguish. The arc voltage continues to drop smoothly (BO path).

Models 1 and 4 are not able to simulate the third part so the response of models 1 and 4 are different from the actual performance of arc furnace. From the results of simulation, we conclude that the models 2 and 3 are more accurate present better results.

Purposed method in this paper, use the active part of the characteristics for arc furnace flicker simulation. This idea implemented for model 1 by the variation of the active arc resistance $R_{2}$.Figures 17 and 19 show the V-I characteristic with sinusoidal change in $R_{1}, R_{2}$ respectively. Comparing the voltage waveform at PCC for modulation by using $R_{1}$, and $R_{2}$ as the modulation coefficient in simulation (Fig 18, 20), we can conclude that using R2 modulation to generate the dynamic response of the furnace, generate more flicker and the results seems to be closer to the real one. As more power is taken by the furnace in part $\mathrm{AB}$ of the VIC, more flicker is expected and using R1 modulation leads us to a less generated flicker case which is not correct.

\section{REFERENCES}

[1] Tongxin Zheng, ElhamB.Makram, Adly A.Girgis. "Effect of Different Arc Furnace Models on Voltage Distortion", IEEE 1998

[2] Wu Ting, Song Wennan, Zhang Yao, "A New Frequency Domain Method for the Harmonic Analysis of power system with Arc Furnace", proceeding of the $4^{\text {th }}$ international conference on advances in power system control, 1997

[3] Omer Ozgun, Ali Abur, "Development of an Arc Furnace Model for Power Quality Studies", IEEE, 1999

[4] Omer Ozgun, Ali Abur, "Flicker Study Using a Novel Arc Furnace Model", IEEE transaction on power delivery Vol.17, No.4, 2002

[5] Srinivas Varadan, Elham B.Makram, AdlyA.Girgis, "A New Time Domain Voltage Source Model for an Arc Furnace Using EMTP", IEEE Transactions On power del. Vol.11, No.3, 1996

[6] C.Sharmeela, G.uma, M.R.Mohan, K.Karthikeyan, "Voltage Flicker Analysis and Mitigation Case Study in ac Electric Arc Furnace Using PSCAD/EMTDC", international conference on power system technology, IEEE 2004

[7] Tongxin Zheng, Elham B.Makram, "An Adaptive Arc Furnace Model", IEEE Transaction on power delivery, vol.15, No.3, july 2000

[8] Gian Carlo Montanari, Mauro loggini, Luco pitti,Enrico Tironi, " The Effects of Series Inductors for Flicker Reduction in Electric Power System Supplying Arc Furnaces", IEEE 1993

[9] G.C.Maontanari, M.loggini, A.carallini, L.pitti, "Flicker and distortion compensation in electrical plants supplying arc furnace", IEEE 1994

[10] G.C.Maontanari, M.loggini, A.carallini, L.pitti, D.Zaninelli, "Arc furnace model for the study of flicker compensation in electrical networks", IEEE Trans. On power delivery, vol3, no.4, 1994

[11] Le tang, Sharma kolluri, Mark, F.MC.Granaghan, "voltage flicker prediction for two simultaneously operated ac arc furnaces",IEEE 1996

[12] Bostjan Blazic,Igor papic, "Analysis of flicker mitigation in utility distribution network", IEEE 2003

[13] S.R.mendis, M.T.Bishop, J.F.Witte, "investigation of voltage flicker in electric arc furnace power system", IEEE 1994 